\title{
Numerical solution of boundary integral equations by means of attenuation factors
}

\author{
Michèle REIFEnBerg AND JEAN-PAUl BERRUT ${ }^{\dagger}$ \\ Institut de Mathématiques, Université de Fribourg, Pérolles, CH-1700 Fribourg, \\ Switzerland
}

[Received 26 June 1997 and in revised form 10 November 1998]

\begin{abstract}
We consider first-kind boundary integral equations with logarithmic kernel such as those arising from solving Dirichlet problems for the Laplace equation by means of single-layer potentials. The first-kind equations are transformed into equivalent equations of the second kind which contain the conjugation operator and which are then solved with a degeneratekernel method based on Fourier analysis and attenuation factors. The approximations we consider, among them spline interpolants, are linear and translation invariant. In view of the particularly small kernel, the linear systems resulting from the discretization can be solved directly by fixed-point iteration.
\end{abstract}

Keywords: boundary integral equations; attenuation factors; Fourier methods; interpolation operators.

\section{Introduction}

Let $\Gamma$ be a Jordan curve and denote by $G$ the interior of $\Gamma$. Our aim is the numerical solution of the boundary integral equation with logarithmic kernel

$$
\frac{1}{2 \pi} \int_{\Gamma} \log |z-w| v(w) \mathrm{d} \sigma(w)=k(z), \quad z \in \Gamma,
$$

where $\sigma$ is an appropriate measure on $\Gamma$. Such an equation arises, for example, when one seeks the solution of the interior Dirichlet problem

$$
\begin{aligned}
& \Delta U=0, \text { in } G, \\
& U=-k, \text { on } \Gamma,
\end{aligned}
$$

as a single-layer potential

$$
U(z):=-\frac{1}{2 \pi} \int_{\Gamma} \log |z-w| v(w) \mathrm{d} \sigma(w), \quad z \in \bar{G} .
$$

Indeed, (1.2) is satisfied independently of the choice of the density function $v$ and, for the boundary condition (1.3) to be satisfied, $v$ has to be a solution of equation (1.1). To ensure

\footnotetext{
† Author to whom correspondence should be addressed.

Email: mreifenbe@sairgroup.ch and jean-paul.berrut@unifr.ch
} 
that $U(z)$ is continuous for $z \rightarrow \Gamma$, and that all arising functions can be written as their Fourier series, we suppose that $v \in \mathrm{L}_{p}(\Gamma)$ for some $p \geqslant 2$, and that $\Gamma$ is a piecewise regular $C^{1}$-Jordan curve (Gaier, 1976).

For the theoretical study of (1.1), as well as for some efficient numerical solution methods, it is useful to split the singular kernel into a singular part independent of the curve and a regular part. Up until 1975, this splitting was performed in a way that destroyed the periodicity, namely as

$$
\log |z(t)-z(s)|=\log |t-s|+\log \left|\frac{z(t)-z(s)}{t-s}\right|
$$

(see Hsiao et al., 1980). Then, Henrici suggested using the kernel for the circle as the denominator, giving

$$
\log |z(t)-z(s)|=\log \left|\mathrm{e}^{\mathrm{i} t}-\mathrm{e}^{\mathrm{i} s}\right|+\log \left|\frac{z(t)-z(s)}{\mathrm{e}^{\mathrm{i} t}-\mathrm{e}^{\mathrm{i} s}}\right|
$$

(see Henrici, 1979; Reichel, 1984, 1986, independently came up with the same idea), and used it to analytically solve the equation for the ellipse. In 1975 also, the second author of the present article (Berrut, 1976) and Reichel independently suggested using this splitting in the numerical solution of (1.1) with trigonometric polynomials for general curves (see Henrici, 1986). The method in Berrut (1976) merely uses one-dimensional FFTs and solves the resulting systems of equations by iteration (see also Berrut, 1986). Many closely related methods have been suggested since (Hoidn, 1983; Arnold, 1983; Lamp et al., 1985; Atkinson, 1988). A theoretical study of the method for $\mathrm{C}^{\infty}$-curves was performed in McLean (1986) and McLean et al. (1989).

Starting with Hsiao et al. (1980), the splitting (1.4) was subsequently used by several authors for the theoretical study of equation (1.1) (see, e.g., (Graham \& Yan, 1990; Sloan, 1992, and the literature cited therein, and for example Yan \& Sloan, 1988, for polygonal curves). In Hsiao et al. (1980), the existence of a Fredholm equation of the second kind derived from (1.1) was first noticed. The kernel and inhomogeneity of this equation were given in Berrut (1986), where the latter equation was solved with the trigonometric degenerate-kernel method of Berrut (1976). The advantage of this kernel, as compared with the Neumann kernel of the double-layer potential (Henrici, 1986), is its smaller size in many examples, resulting in a faster convergence of the direct application of fixed-point iteration (see the numerical example in Berrut, 1986).

Other effective methods for solving (1.1) have been presented, among them qualocation methods (see Chandler \& Sloan, 1990; Sloan \& Burn, 1992; Sarane \& Sloan, 1992, for smooth curves and Elschner \& Graham, 1994, for curves with corners). Another efficient method for piecewise analytic curves was developed in Hough (1990), where a very elaborate program package was also given.

The structure of the paper is as follows. After a short reminder in Section 2.1 on the theoretical analysis of (1.1), the derivation of the equivalent equation of the second kind mentioned above is outlined in Section 2.2. Since it contains the conjugation operator it is best solved by Fourier methods. The case where $\Gamma$ is an open arc instead of a closed curve is briefly considered in Section 2.3. In Section 3 we present a new degeneratekernel Fourier method which makes use of the theory of attenuation factors and covers 
a large class of approximation operators, namely all the linear and translation invariant ones, like trigonometric and spline interpolation operators. Finally, some convergence and computational results are given in Sections 4 and 5.

\section{The integral equations}

Denote by $I$ the interval $[0,2 \pi] \subset \mathbb{R}$, by $I^{2}$ the square $[0,2 \pi] \times[0,2 \pi] \subset \mathbb{R}^{2}$ and let $\Gamma: z=z(t), t \in I$, be a Jordan curve. If $z \in \mathrm{C}^{\infty}, \Gamma$ is said to be smooth. $\Gamma$ is called a piecewise regular $\mathrm{C}^{r}$-Jordan curve if there exists a partition of $I$ into a finite number of subintervals $\left[t_{i}, t_{i+1}\right], i=0, \ldots, p-1$, with $0=t_{0}<t_{1}<\cdots<t_{p-1}<t_{p}=2 \pi$, such that the restriction of the parametrization $z(t)$ to each subinterval is $r$ times continuously differentiable with $z^{\prime}(t) \neq 0$. The points $z\left(t_{i}\right), i=0, \ldots, p-1$, are called corners. We denote by $\epsilon(\Gamma)$ the set of nodes corresponding to the corners of $\Gamma$. A regular $\mathrm{C}^{r}$-Jordan curve is a piecewise regular $\mathrm{C}^{r}$-Jordan curve without corners.

Using the notation $x(t):=v(z(t))\left|z^{\prime}(t)\right|$ and $f(t):=k(z(t))$, equation (1.1) reads

$$
\frac{1}{2 \pi} \int_{0}^{2 \pi} \log |z(t)-z(s)| x(s) \mathrm{d} s=f(t), \quad t \in I
$$

or in operator notation

$$
\mathrm{A} x=f .
$$

\subsection{Decomposition of the operator $A$ and subsequent theoretical results}

As explained in the introduction, we shall decompose the logarithmic kernel of the operator A into the sum of a singular kernel and a bounded one (Berrut, 1986)

$$
\log |z(t)-z(s)|=q(t, s)+r(t, s),
$$

where

$$
q(t, s):=\log \rho\left|\mathrm{e}^{\mathrm{i} t}-\mathrm{e}^{\mathrm{i} s}\right|, \quad r(t, s):=\log \left|\frac{z(t)-z(s)}{\rho\left(\mathrm{e}^{\mathrm{i} t}-\mathrm{e}^{\mathrm{i} s}\right)}\right|
$$

and $\rho \in \mathbb{R}^{+} \backslash\{1\}$. This means that we subtract from the kernel of A the corresponding kernel for the circle of radius $\rho$. This decomposition splits the operator A into the sum of two operators,

$$
\mathrm{A}=\mathrm{Q}+\mathrm{R},
$$

with $\mathrm{Q}:=(\operatorname{Int} q), \mathrm{R}:=(\operatorname{Int} r)$, where

$$
(\operatorname{Int} h) x(t):=\frac{1}{2 \pi} \int_{0}^{2 \pi} h(t, s) x(s) \mathrm{d} s .
$$

(We have borrowed this notation from Halmos \& Sunder (1978): it has the advantage of simply allowing for composite kernels like those appearing in the following sections.) For 
$h \in \mathrm{L}_{2}\left(I^{2}\right)$, (Int $\left.h\right)$ is a linear, bounded and compact operator on $\mathrm{L}_{2}(I)$ called a HilbertSchmidt integral operator (H-S operator).

The analysis of the operators $\mathrm{Q}$ and $\mathrm{R}$ is carried out in $2 \pi$-periodic Sobolev spaces. Let $\mathrm{H}^{m}(I)$ be the periodic Sobolev space of order $m$ and denote by $\|\cdot\|_{m}$ the corresponding Sobolev norm. We denote the two-dimensional tensor products of such spaces by $\mathrm{H}^{(m, n)}\left(I^{2}\right)$. In two dimensions we consider also the space $\mathrm{C}_{p}^{m}\left(I^{2}\right)$ of all $m$ times continuously differentiable functions on $\mathbb{R}^{2}$ that are $2 \pi$-periodic in both variables. Then the two-dimensional $2 \pi$-periodic Sobolev space $\mathrm{H}^{m}\left(I^{2}\right)$ is defined as the completion of $C_{p}^{\infty}\left(I^{2}\right):=\bigcap_{j} C_{p}^{j}\left(I^{2}\right)$ with respect to the Sobolev norm

$$
\|h\|_{m}:=\left(\sum_{k, \ell=-\infty}^{+\infty}\left(1+k^{2}+\ell^{2}\right)^{m}\left|c_{k \ell}(h)\right|^{2}\right)^{1 / 2},
$$

where $c_{k \ell}(h)$ denotes the $(k, \ell)$ th two-dimensional Fourier coefficient of $h$.

By analysing the Fourier series of $\mathrm{Q} x$, which can be given explicitly, one can easily see that $\mathrm{Q}$ is a bounded operator from $\mathrm{H}^{p}(I)$ to $\mathrm{H}^{p+1}(I)$ for any $p \in \mathbb{R}$ (Sloan, 1992). The inverse operator $\mathrm{Q}^{-1}$ is a bounded operator from $\mathrm{H}^{p+1}(I)$ to $\mathrm{H}^{p}(I)$ and can be written in terms of the differentiation operator $\mathrm{D}$ and the conjugation operator $\mathrm{K}$ as (Berrut, 1986)

$$
\mathrm{Q}^{-1}: y \in \mathrm{H}^{p+1}(I) \rightarrow \mathrm{Q}^{-1} y=\frac{c_{0}(y)}{\log \rho}-2 \mathrm{KD} y=\mathrm{J} y-2 \mathrm{KD} y \in \mathrm{H}^{p}(I),
$$

where $\mathrm{J} y:=c_{0}(y) / \log \rho$. The conjugation operator $\mathrm{K}$ (or Hilbert transform on the circle) is the linear and bounded operator from $\mathrm{L}_{2}(I)$ into itself which maps

$$
x(t)=\sum_{n=-\infty}^{+\infty} c_{n}(x) \mathrm{e}^{\mathrm{i} n t} \in \mathrm{L}_{2}(I)
$$

to

$$
\mathrm{K} x(t)=\sum_{n=-\infty}^{+\infty}-\mathrm{i} \operatorname{sign}(n) c_{n}(x) \mathrm{e}^{\mathrm{i} n t}, \quad(\operatorname{sign}(0):=0) .
$$

Let us now look at the operators $\mathrm{R}$ and $\mathrm{DR}$ and list some existence/uniqueness results. If $\Gamma$ is a piecewise analytic $\mathrm{C}^{1}$-curve or a piecewise $\mathrm{C}^{2}$ regular $\mathrm{C}^{1}$-curve, then (Hoidn, 1983) $\mathrm{D} r$ is bounded in a neighbourhood of $\left(t_{i}, t_{i}\right), t_{i} \in \epsilon(\Gamma)$, from which it follows that $\mathrm{D} r$ belongs to $\mathrm{L}_{2}\left(I^{2}\right)$ and $\mathrm{DR}: \mathrm{L}_{2}(I) \rightarrow \mathrm{L}_{2}(I)$ is a Hilbert-Schmidt operator, and is therefore compact. With (2.3) we can then write $\mathrm{A}$ as $\mathrm{Q}+\mathrm{R}=\mathrm{Q}\left(\mathrm{I}+\mathrm{Q}^{-1} \mathrm{R}\right)=\mathrm{Q}(\mathrm{I}-2 \mathrm{KDR}+\mathrm{JR})$. Using the fact that A is injective if the logarithmic capacity $\gamma$ of $\Gamma$ is different from one (Yan \& Sloan, 1988), it follows with the Fredholm alternative that for each Jordan curve $\Gamma$ with $\gamma \neq 1$ and such that $\mathrm{D} r \in \mathrm{L}_{2}\left(I^{2}\right)$

$$
\text { A: } \mathrm{H}^{0}(I) \rightarrow \mathrm{H}^{1}(I) \text { is an isomorphism. }
$$

If $\Gamma$ is a polygon or an arbitrary piecewise regular $\mathrm{C}^{2}$-Jordan curve without cusps, then $\mathrm{D} r$ is not bounded in a neighbourhood of $\left(t_{i}, t_{i}\right), t_{i} \in \epsilon(\Gamma)$. The first author has shown (Reifenberg, 1997) that $\mathrm{D} r$ no longer belongs to $\mathrm{L}_{2}\left(I^{2}\right)$ (in fact, $\mathrm{D} r \in \mathrm{H}^{-\varepsilon}\left(I^{2}\right), \varepsilon>0$ ) 
and thus that DR can no longer be guaranteed to be a compact operator on $\mathrm{H}^{0}(I)$. For the case of a polygon with a parametrization proportional to arclength it has been shown (Yan \& Sloan, 1988) that DR $=\mathrm{C}+\mathrm{B}$, where $\mathrm{C}$ is a compact operator on $\mathrm{H}^{0}(I)$ and $\mathrm{B}$ is a non-compact Mellin convolution operator local to each corner. It follows that, for such $\Gamma$, A is still an isomorphism from $\mathrm{H}^{0}(I)$ to $\mathrm{H}^{1}(I)$. With the help of perturbation arguments, Elschner \& Graham (1995) extend this result to more general parametrizations. The generalization to arbitrary piecewise analytic Jordan curves without cusps or even to piecewise regular $\mathrm{C}^{2}$-Jordan curves without cusps should be possible.

On the other hand, if we restrict $\Gamma$ to a smooth curve, then more general results hold. Here the kernel $\operatorname{Dr}(t, s)$ is a $\mathrm{C}^{\infty} 2 \pi$-periodic function in both variables. Thus DR : $\mathrm{H}^{m}(I) \rightarrow \mathrm{H}^{m}(I)$ is compact $\forall m \in \mathbb{R}$ (Yan \& Sloan, 1988), and we conclude that, if $\gamma \neq 1, \mathrm{~A}$ is an isomorphism from $\mathrm{H}^{m}(I)$ to $\mathrm{H}^{m+1}(I)$.

\subsection{An operator equation of the second kind}

In constrast to most other authors (who solve (2.1) with Galerkin, collocation or qualocation methods) we want to use a degenerate-kernel method. Since (2.1) is not directly amenable to such a method, we first transform it through multiplication by $\mathrm{Q}^{-1}$ into the equivalent equation of the second kind

$$
\left(\mathrm{I}+\mathrm{Q}^{-1} \mathrm{R}\right) x=\mathrm{Q}^{-1} f .
$$

For a function of two variables $h \in \mathrm{L}_{2}\left(I^{2}\right)$ with the Fourier series

$$
\sum_{n, m=-\infty}^{+\infty} c_{n m}(h) \mathrm{e}^{\mathrm{i} n t} \mathrm{e}^{-\mathrm{i} m s}
$$

we define the conjugate function with respect to the first variable (again denoted by K) by

$$
\mathrm{K} h(t, s)=\sum_{n, m=-\infty}^{+\infty}-\mathrm{i} \operatorname{sign}(n) c_{n m}(h) \mathrm{e}^{\mathrm{i} n t} \mathrm{e}^{-\mathrm{i} m s} .
$$

One then verifies that $\mathrm{KDR}=\mathrm{KD}(\mathrm{Int} r)=(\mathrm{Int} \mathrm{KD} r)$, where $\mathrm{D} r$ is the derivative of $r$ with respect to the first variable (Berrut, 1986):

$$
\operatorname{Dr}(t, s)=\frac{\partial}{\partial t} \log \left|\frac{z(t)-z(s)}{\mathrm{e}^{\mathrm{i} t}-\mathrm{e}^{\mathrm{i} s}}\right|=\operatorname{Re}\left\{\frac{z^{\prime}(t)}{z(t)-z(s)}-\frac{\mathrm{ie}^{\mathrm{i} t}}{\mathrm{e}^{\mathrm{i} t}-\mathrm{e}^{\mathrm{i} s}}\right\} .
$$

Using (2.3) we obtain the operator equation of the second kind (Berrut, 1986)

$$
x-2(\operatorname{Int} \mathrm{KD} r) x+\frac{c_{0}((\operatorname{Int} r) x)}{\log \rho}=\frac{c_{0}(f)}{\log \rho}-2 \mathrm{KD} f .
$$

In contrast with the integral equation of the first kind (2.1), (2.7) no longer contains a singular logarithmic kernel but the better behaved kernel $\mathrm{D} r$. Moreover, numerical calculations (Berrut, 1985) show that in most cases the $\mathrm{L}_{2}$ and $\mathrm{L}_{\infty}$-norms of this kernel are smaller than those of the Neumann kernel of the second-kind equation obtained from 
the double-layer representation of the solution (Henrici, 1986). (For instance, for the disc of radius $\rho$, one has $\mathrm{D} r=0$, whereas Neumann's kernel is $1 / 2 \pi \rho$, (see Henrici, 1986, p 283).) Also, all the eigenvalues of the integral operator are smaller than one (Stenger $\&$ Schmidtlein, 1998). As a consequence, direct application of fixed-point iteration to the discretized equation (see Berrut, 1986, and Section 3) always converges as soon as $N$ is large enough for a good approximation of the kernel, and typically in a small number of iterations. Numerical experience with the Dirichlet problem of conformal mapping (see Example 2, Section 5) also demonstrates that, for domains with reentrant corners or similar smooth domains like reflected ellipses with small parameters (Berrut, 1985), the solution of the discretized equation is more accurate than that obtained with the Neumann kernel equation (called Warschawski's equation in that context (Henrici, 1986)).

\subsection{Open arcs}

We can also consider the case where $\Gamma$ is no longer a closed curve, but an open arc parametrized by $v(\tau), \tau \in[-1,1]$, with $\left|v^{\prime}(\tau)\right|>0$. After transforming it to the circle with the change of variable $t=\cos ^{-1} \tau$ (Yan \& Sloan, 1988) and after defining $z(t):=v(\cos t)$, $t \in[0, \pi]$, equation (1.1) becomes

$$
\frac{1}{2 \pi} \int_{0}^{\pi} \log |z(t)-z(s)| v(z(s))\left|v^{\prime}(\cos s)\right| \sin s \mathrm{~d} s=k(z(t)), \quad t \in[0, \pi] .
$$

Let $x(t):=\frac{1}{2} v(z(t))|\sin t|\left|v^{\prime}(\cos t)\right|$ and $f(t):=k(z(t))$. Then $x$ and $f$ are even, $2 \pi-$ periodic functions and (2.8) can be written as

$$
\frac{1}{\pi} \int_{0}^{\pi} \log |z(t)-z(s)| x(s) \mathrm{d} s=f(t), \quad t \in[0, \pi] .
$$

Moreover, the integral in the above expression can be replaced by one half of the integral over a full period; we then again get (2.2) and we can solve it by the same numerical methods.

The solution of (2.8) as a function of that of (1.1) is given by

$$
v(z)=v(z(t))=\frac{2 x(t)}{\left|v^{\prime}(\cos t)\right| \sqrt{1-\left(v^{-1}(z)\right)^{2}}} .
$$

Due to the factor $\sqrt{1-\left(v^{-1}(z)\right)^{2}}$ it has a singularity at the two extremities of the contour, even if $x$ is smooth, which reflects the different character of the solutions on closed and open contours.

The theoretical analysis has been given by Yan \& Sloan (1988). All the operators are considered in even Sobolev spaces $\mathrm{H}_{e}^{p}:=\left\{x \in \mathrm{H}^{p} \mid c_{-n}(x)=c_{n}(x) \forall n\right\}$ and equation (2.9) is split into

$$
\left(\mathrm{Q}_{e}+\mathrm{R}_{e}\right) x=f
$$


with

$$
\mathrm{Q}_{e} x(t):=\frac{1}{\pi} \int_{0}^{\pi} \log |\rho(\cos t-\cos s)| x(s) \mathrm{d} s
$$

and

$$
\mathrm{R}_{e} x(t):=\frac{1}{\pi} \int_{0}^{\pi} \log \left|\frac{z(t)-z(s)}{\rho(\cos t-\cos s)}\right| x(s) \mathrm{d} s,
$$

for $\rho \neq 1$. The kernel $r_{e}(t, s):=\log |(z(t)-z(s)) /(\rho(\cos t-\cos s))|$ is $2 \pi$-periodic and even in each variable and has the same properties as $r$. For an even function $x$ one has $c_{n}(x)=c_{-n}(x)$ for all $n$, from which the cosine Fourier series representation of $x$

$$
x(t)=a_{0}(x)+\sum_{n=1}^{\infty} a_{n}(x) \cos n t
$$

follows, where $a_{n}(x)=c_{n}(x)+c_{-n}(x)=2 c_{n}(x)$. Making use of the fact that $\mathrm{Q}_{e} x=$ $\mathrm{Q} x$ for $x$ even, we easily obtain the cosine Fourier series of $\mathrm{Q}_{e} x$, from which it follows immediately that $\mathrm{Q}_{e}$ is a bounded and invertible operator from $\mathrm{H}_{e}^{p}$ to $\mathrm{H}_{e}^{p+1}$ for every $p \in \mathbb{R}$. Consequently, (2.9) is equivalent to the equation of the second kind

$$
\left(\mathrm{I}+\mathrm{Q}_{e}^{-1} \mathrm{R}_{e}\right) x=\mathrm{Q}_{e}^{-1} f .
$$

For $y$ even we still have $\mathrm{Q}_{e}^{-1} y=c_{0}(y) / \log \rho-2 \mathrm{KD} y$ and thus (2.10) can be written as

$$
x-2\left(\operatorname{Int}_{e} \mathrm{KD}_{e}\right) x+\frac{c_{0}\left(\left(\operatorname{Int}_{e} r_{e}\right) x\right)}{\log \rho}=\frac{c_{0}(f)}{\log \rho}-2 \mathrm{KD} f,
$$

which corresponds to (2.7) with $r$ replaced by $r_{e}$ and Int replaced by Int $_{e}$, where

$$
\left(\operatorname{Int}_{e} h\right) x(t):=\frac{1}{\pi} \int_{0}^{\pi} h(t, s) x(s) \mathrm{d} s .
$$

If $x$ is even then (2.11) is equivalent to (2.7).

\section{The numerical method}

In this section we describe the numerical solution of the equation of the second kind (2.7) by a degenerate-kernel method making use of attenuation factors. In order to simplify the notation, we shall from now on set $\rho:=$ e, i.e., $\log \rho:=1$.

Let $\left\{t_{k}\right\}, t_{k}:=k 2 \pi / N, k=0, \ldots, N-1$, be the equidistant mesh on $I$ and $\left\{\left(t_{k}, s_{\ell}\right)\right\}$, $\left(t_{k}, s_{\ell}\right):=(k 2 \pi / N, \ell 2 \pi / N), k, \ell=0, \ldots, N-1$, be the equidistant mesh on $I^{2}$. For a $2 \pi$-periodic function $f \in \mathrm{L}_{2}(I)$ defined everywhere, we denote by $\tilde{f}$ the $N$-periodic biinfinite real sequence $\left\{f_{k}\right\}_{k=-\infty}^{+\infty}$, where $f_{k}:=f\left(t_{k}\right), k=0, \ldots, N-1$, and $f_{k+N}=f_{k}$

for all $k$. Similarly, for a function of two variables $h$, we define $\widetilde{h}:=\left\{h_{k, \ell}\right\}_{k, \ell=-\infty}^{+\infty}$, where $h_{k, \ell}:=h\left(t_{k}, s_{\ell}\right), k, \ell=0, \ldots, N-1$, and where the other values are obtained through 
$N$-periodic repetition in $k$ and $\ell$. As in Henrici (1986), we denote the space of such one-, respectively two-dimensional sequences by $\Pi_{N}$, resp. $\Pi_{N}^{(2)}$. We approximate $f$ and $h$ by $\widehat{f}:=\mathrm{P} \widetilde{f}$, respectively $\widehat{h}:=\mathbf{P} \tilde{h}$, where $\mathrm{P}: \Pi_{N} \rightarrow \Pi$ and $\mathbf{P}: \Pi_{N}^{(2)} \rightarrow \Pi^{(2)}$ are linear and translation invariant approximation operators, $\Pi$ and $\Pi^{(2)}$ denoting the spaces of $2 \pi$-periodic real one-, respectively two-dimensional functions. (Since all functions are $2 \pi$-periodic, characterizing approximations by a ' , does not create any confusion with the Fourier transform.) These operators have the property

$$
c_{n}(\mathrm{P} \tilde{f})=\tau_{n} \widehat{c}_{n}(f) \quad \text { for all } \quad n \in \mathbb{Z}, \tilde{f} \in \Pi_{N}, f \in \Pi,
$$

where $c_{n}(x)$ stands for the $n$th exact, $\widehat{c}_{n}(x)$ for the $n$th discrete Fourier coefficient of $x$ and $\tau_{n}$ is the $n$th attenuation factor corresponding to the operator P. Thus $\mathrm{P} \widetilde{f}=$ $\sum_{n=-\infty}^{\infty} \tau_{n} \widehat{c}_{n}(f) \mathrm{e}^{\mathrm{i} n t}$. If $\mathrm{P}$ has the additional property

$$
\mathrm{P}(\{\ldots, 1,1,1, \ldots, 1, \ldots\})=1,
$$

and if

$$
\text { P preserves central symmetry, }
$$

then $\tau_{0}=1, \tau_{j N}=0$ for all $j \in \mathbb{Z}^{*}:=\mathbb{Z} \backslash\{0\}$ and $\tau_{-n}=\tau_{n} \in \mathbb{R}$ for all $n \in \mathbb{Z}$ (see Gautschi, 1972).

Moreover, if $\mathbf{P}$ is the tensor-product of $\mathbf{P}$ by itself, one has

$$
c_{n m}(\mathbf{P} \tilde{h})=\tau_{n} \tau_{m} \widehat{c}_{n m}(h),
$$

where $c_{n m}(h)$ and $\widehat{c}_{n m}(h)$ are respectively the $(n, m)$ th two-dimensional exact and discrete Fourier coefficients of $h$ (Gutknecht, 1987).

Setting $\mathrm{T}:=\mathrm{Q}^{-1} \mathrm{R}$ and $g:=\mathrm{Q}^{-1} f$, equation (2.5) reads

$$
x+\mathrm{T} x=g .
$$

In Berrut \& Reifenberg (1997) we solved Fredholm integral equations of the second kind $x+\mathrm{H} x=f$ by approximating the operator $\mathrm{H}:=(\operatorname{Int} h)$ for $h \in \mathrm{L}_{2}\left(I^{2}\right)$ by $\widehat{\mathrm{H}}:=(\operatorname{Int} \widehat{h})=$ (Int $\mathbf{P} \widetilde{h}$ ) and the inhomogeneity $f \in \mathrm{L}_{2}(I)$ by $\widehat{f}=\mathrm{P} \widetilde{f}$. Applying this to equation (3.5) would mean approximating (Int KDr $)$ by (Int $\widehat{\mathrm{KD} r} r)=(\operatorname{Int} \mathbf{P K D} r)$ and $\mathrm{KD} f$ by $\widehat{\mathrm{KD} f}=$ $\widetilde{\mathrm{PND} f}$. To achieve these approximations we would have to compute the discrete Fourier coefficients of $\mathrm{KD} r$ and $\mathrm{KD} f$, which would require knowledge of the values of $\mathrm{KD} r$ at the $N^{2}$ points $\left(t_{k}, s_{\ell}\right), k, \ell=0, \ldots, N-1$, and of $\operatorname{KD} f$ at the points $t_{k}$. But, except for special curves such as the ellipse and the circle, these values are not available: one cannot obtain the values $\operatorname{KDr}\left(t_{k}, s_{\ell}\right)$ and $\operatorname{KD} f\left(t_{k}\right)$ from those of $\mathrm{Dr}$ and $\mathrm{D} f$. For this reason we first approximate the known functions $r, \mathrm{D} r, f$ and $\mathrm{D} f$ by $\widehat{r}=\mathbf{P} \widetilde{r}, \widehat{\mathrm{D} r}=\mathbf{P D} r, \widehat{f}=\mathrm{P} \widetilde{f}$ and $\widetilde{\mathrm{D} f}=\mathrm{PDf}$, respectively, and we conjugate afterwards, making use of formulas (2.4) and (2.6). This yields the operator

$\widehat{\mathrm{T}}: x \in \mathrm{L}_{2}(I) \rightarrow \widehat{\mathrm{T}} x:=-2(\operatorname{Int} \mathrm{K} \widehat{\mathrm{D} r}) x+c_{0}((\operatorname{Int} \widehat{r}) x)=\mathrm{K}(-2(\operatorname{Int} \widehat{\mathrm{D} r}) x)+c_{0}((\operatorname{Int} \widehat{r}) x)$

as an approximation of $\mathrm{T}$, and the approximate inhomogeneity

$$
\widehat{g}=-2 \mathrm{KDf}+c_{0}(\widehat{f}) \text {. }
$$


We observe that $\widehat{T} x$ and $\widehat{g}$ are both in the form K $\widehat{y}$ for some function $y \in \mathrm{L}_{2}^{0}(I)$, plus some constant term, where $\mathrm{L}_{2}^{0}(I)$ is the subspace of those functions in $\mathrm{L}_{2}(I)$ whose zeroth Fourier coefficient equals 0 . Therefore the same is true for the solution $\widehat{x}$ of the approximate equation

$$
\widehat{x}+\widehat{T} \widehat{x}=\widehat{g}
$$

and we have the following theorem.

THEOREM 3.1 The solution of (3.8), if it exists, can be expressed as

$$
\widehat{x}=\mathrm{KP} \tilde{y}+\alpha
$$

for some $\tilde{y} \in \Pi_{N}$ and some $\alpha \in \mathbb{C}$. Moreover, with $\widehat{y}:=\mathrm{P} \tilde{y}$, (3.8) can be written explicitly as

$$
\begin{aligned}
\mathrm{K} \widehat{y}+\alpha-2(\operatorname{Int} \mathrm{K} \widehat{\mathrm{Dr}}) \mathrm{K} \hat{y}+c_{0}((\operatorname{Int} \widehat{r}) \mathrm{K} \widehat{y}) & -2(\operatorname{Int} \mathrm{K} \widehat{\mathrm{Dr}}) \alpha+c_{0}((\operatorname{Int} \widehat{r}) \alpha) \\
& =-2 \mathrm{~K} \widehat{\mathrm{D} f}+c_{0}(\widehat{f}) .
\end{aligned}
$$

Conditions guaranteeing the existence of $\widehat{x}$ will be given in Theorem 4.1. We do not need to know the value of $\widehat{c}_{0}(\widehat{y})$ since we apply the conjugate operator $\mathrm{K}$ to $\widehat{y}$ whenever the latter occurs, which multiplies $\widehat{c}_{0}(\widehat{y})$ by zero. We can therefore set $\widehat{c}_{0}(\widehat{y})=0$. Expanding all the terms of (3.9) into their Fourier series, using the orthogonality of the trigonometric functions as well as the properties (3.1) and (3.4) and equating on both sides the coefficients of $\mathrm{e}^{\mathrm{i} n t}, n \in \mathbb{Z}^{*}$, we obtain the infinite system

$$
\left\{\begin{array}{l}
\alpha-\mathrm{i} \sum_{m=-\infty}^{+\infty} \operatorname{sign}(m) \tau_{m}^{2} \widehat{c}_{0 m}(r) \widehat{c}_{m}(\widehat{y})+\widehat{c}_{00}(r) \alpha=\widehat{c}_{0}(f), \\
\tau_{n} \widehat{c}_{n}(\widehat{y})+2 \mathrm{i} \tau_{n} \sum_{m=-\infty}^{+\infty} \operatorname{sign}(m) \tau_{m}^{2} \widehat{c}_{n m}(\mathrm{D} r) \widehat{c}_{m}(\widehat{y})-2 \tau_{n} \widehat{c}_{n 0}(\mathrm{D} r) \alpha=-2 \tau_{n} \widehat{c}_{n}(\mathrm{D} f) .
\end{array}\right.
$$

For the existence of a unique solution $\left(\alpha, \widehat{c}_{1}(\widehat{y}), \ldots, \widehat{c}_{N-1}(\widehat{y})\right)^{T}$ the infinite system (3.10) should be equivalent to $N$ distinct equations. Making use of the $N$-periodicity of the discrete Fourier coefficients $\widehat{c}_{n m}(\mathrm{Dr})$ and $\widehat{c}_{m}(\widehat{y})$, we get the following finite $\mathrm{N}$ dimensional system for the $N-1$ unknown coefficients $c_{n}(\widehat{y}), n=1, \ldots, N-1$, and $\alpha$ :

$$
\left\{\begin{array}{l}
\alpha-\mathrm{i} \sum_{m=1}^{N-1} \rho_{m} \widehat{c}_{0 m}(r) \widehat{c}_{m}(\widehat{y})+\widehat{c}_{00}(r) \alpha=\widehat{c}_{0}(f), \\
\widehat{c}_{n}(\widehat{y})+2 \mathrm{i} \sum_{m=1}^{N-1} \rho_{m} \widehat{c}_{n m}(\mathrm{D} r) \widehat{c}_{m}(\widehat{y})-2 \widehat{c}_{n 0}(\mathrm{D} r) \alpha=-2 \widehat{c}_{n}(\mathrm{D} f),
\end{array}\right.
$$

where

$$
\rho_{m}:=\sum_{\ell=-\infty}^{+\infty} \operatorname{sign}(m+\ell N) \tau_{m+\ell N}^{2}, \quad \text { for } \quad m=1, \ldots, N-1 .
$$

REMARK 3.1 If $\log \rho \neq 1$, then $\log \rho$ appears merely in the first equation as a factor of the first term $\alpha$. It could be used numerically to balance this equation when its terms are very different in size. 
REMARK 3.2 Instead of solving Symm's integral equation (1.1) or (2.1), Henrici made use of his splitting (1.4) and solved the equation

$$
\mathrm{Q} x=-\mathrm{R} x+f
$$

by developing the given functions and the unknown $x$ into their Fourier series, which yields the corresponding infinite linear system

$$
-\frac{1}{2|n|} c_{n}(x)=-\sum_{m=-\infty}^{+\infty} c_{n m}(r) c_{m}(x)=c_{n}(f), \quad n \neq 0 .
$$

Writing this equation for trigonometric interpolants, as in Berrut (1976), one gets a finite linear system which can be written as an approximate functional equation

$$
\mathrm{Q} \widehat{x}=-\widehat{\mathrm{R}} \widehat{x}+\widehat{f},
$$

for the unknown

$$
\widehat{x}:=\sum_{-N / 2}^{N / 2} " \widehat{c}_{n}(\widehat{x}) \mathrm{e}^{\mathrm{i} n t},
$$

where $\widehat{\mathrm{R}}:=(\operatorname{Int} \mathbf{P} \widetilde{r})$ and $\widehat{f}:=\mathrm{P} \widetilde{f}, \mathrm{P}$ and $\mathbf{P}$ denoting respectively the one- and twodimensional trigonometric interpolation operators. The approximate equation (3.12) looks simpler than the equation of the second kind (2.7). Suppose however that, instead of being trigonometric interpolation operators, $\mathrm{P}$ and $\mathbf{P}$ are spline, respectively tensor product spline interpolation operators. Assuming $\widehat{x}=\mathrm{P} \widetilde{x}$, one sees that $\widehat{\mathrm{R}} \widehat{x}$ is also a spline but unfortunately this is not true for $\mathrm{Q} \widehat{x}$. Hence, one cannot solve the equation corresponding to (3.12) for arbitrary linear and translation invariant approximation operators.

REMARK 3.3 Since the corresponding matrices are large and dense, in practice we do not solve the linear system (3.11) by Gaussian elimination, but we directly apply the fixedpoint iteration described in Berrut \& Reifenberg (1997). This means that at the $(j+1)$ th iteration step, $j=0,1, \ldots$, we compute the values

$$
\left\{\begin{array}{c}
\alpha^{(j+1)}:=\mathrm{i} \sum_{m=1}^{N-1} \rho_{m} \widehat{c}_{0 m}(r) \widehat{c}_{m}\left(\widehat{y}^{(j)}\right)+\widehat{c}_{00}(r) \alpha^{(j)}-\widehat{c}_{0}(f), \\
\widehat{c}_{n}\left(\widehat{y}^{(j+1)}\right):=-\frac{2 \mathrm{i}}{N} \sum_{k=0}^{N-1}\left[\sum_{m=1}^{N-1} \rho_{m} \widehat{c}_{m}\left(\mathrm{D} r^{(k)}\right) \widehat{c}_{m}\left(\widehat{y}^{(j)}\right)\right] w^{-k n} \\
+2 \widehat{c}_{n 0}(\mathrm{D} r) \alpha^{(j)}-2 \widehat{c}_{n}(\mathrm{D} f),
\end{array}\right.
$$

for $n=1, \ldots, N-1$, where $w:=\mathrm{e}^{2 \pi \mathrm{i} / N}$ and where the $\widehat{c}_{m}\left(\mathrm{D} r^{(k)}\right)$ are the coefficients of the trigonometric polynomial interpolating the function $\operatorname{Dr}^{(k)}(s):=\operatorname{Dr}\left(t_{k}, s\right)$ between the points $s_{\ell}, \ell=0, \ldots, N-1$. These values are the same at every interpolation step and therefore are computed only once through $N$ one-dimensional fast Fourier transforms (FFTs) in about $\frac{1}{2}\left(N^{2} \log N\right)$ (complex) flops. (For real functions the practical computations are obviously more efficient in real arithmetic, see Berrut (1985).) The coefficients $\widehat{c}_{0 m}(r), m=0, \ldots, N-1$, can be calculated simultaneously by a single 
one-dimensional FFT, so that this iterative method does not require any two-dimensional FFTs. If $N$ is a power of 2 then the total number of flops needed for the FFTs is about $\frac{1}{2}(N+M+1) N \log _{2} N$, where $M$ denotes the number of iterations. Since the matrixvector products require $M N^{2}$ flops, for $N$ large the iterative solution is faster than Gaussian elimination as long as the number of iterations is less than about $N / 3$. The iterative method is equivalent to that using the two-dimensional FFT, and converges as soon as $N$ is large enough for a good approximation of the kernel of the integral operator.

REMARK 3.4 Let us come back to the case where $\Gamma$ is an open arc and consider the approximate equation

$$
\widehat{x}+\widehat{\mathrm{T}_{e}} \widehat{x}=\widehat{g},
$$

where $\widehat{\mathrm{T}}_{e}$ is defined as $\widehat{\mathrm{T}}$ in (3.6) with $r$ replaced by $r_{e}$ and Int replaced by Int ${ }_{e}$. Considering that $\widehat{f}$ is even (odd) if $f$ is even (odd), and that the derivative and the conjugate function of an even, respectively odd function are odd, respectively even, we see that for $x$ even, $\widehat{\mathrm{T}}_{e} x$ and $\widehat{g}$ are both of the form $K \widehat{y}$ plus a constant term, where $\widehat{y}$ is an odd function in $\mathrm{L}_{2}^{0}$. We thus set $\widehat{x}:=\mathrm{K} \hat{y}+\alpha$ for an odd function $\hat{y} \in \mathrm{L}_{2}^{0}$ and $\alpha \in \mathbb{C}$. Since all the functions involved in the approximate equation are either even or odd, expansions in cosine, respectively sine series instead of exponential Fourier series can be used. The resulting system is then equivalent to (3.10).

\subsection{Examples of the use of some interpolation operators}

We derive here formulas for $\rho_{m}$ in some of the most important cases. Indeed, the presence of the sign function in $\rho_{m}$ makes them all quite different from the corresponding expressions for the general Fredholm equation in Berrut \& Reifenberg (1997). The same is true also for the evaluation of the approximate solution in the following paragraph.

EXAMPLE 1 Trigonometric interpolation

Here only a finite number of attenuation factors are non-zero and the factors $\rho_{m}$ can be computed in an easy way. They are given by $\rho_{m}=1$ if $1 \leqslant m<N / 2, \rho_{m}=0$ if $m=0$ or $N / 2$ and $\rho_{m}=-1$ if $N / 2<m \leqslant N-1$. The method is then equivalent to that in Berrut (1986).

\section{EXAMPLE 2 Spline interpolation}

(a) Spline interpolants of even order $2 d(d \geqslant 1)$.

Since

$$
\tau_{m+\ell N}=\left(\frac{m}{\ell N+m}\right)^{2 d} \tau_{m}
$$

we can write for $m=1, \ldots, N-1$

$$
\rho_{m}=\tau_{m}^{2}\left[-\sum_{\ell=-\infty}^{-1}\left(\frac{m}{\ell N+m}\right)^{4 d}+1+\sum_{\ell=1}^{+\infty}\left(\frac{m}{\ell N+m}\right)^{4 d}\right] .
$$

Let us set as in Gutknecht (1986)

$$
\psi_{p}(z):=\sum_{\ell=1}^{+\infty}\left(\frac{z}{\ell+z}\right)^{p+1} \quad \text { for } z \in \mathbb{C} \backslash\{-1,-2, \ldots\}, p=1,2, \ldots
$$


Then

$$
\rho_{m}=\tau_{m}^{2}\left[-\psi_{4 d-1}\left(-\frac{m}{N}\right)+1+\psi_{4 d-1}\left(\frac{m}{N}\right)\right] .
$$

The function $\psi_{p}(z)$ can be expressed in terms of the polygamma function $\psi^{(p)}(z)$, the $p$ th derivative of the psi function $\psi(z)=(\mathrm{d} / \mathrm{d} z) \log \Gamma(z)=\Gamma^{\prime}(z) / \Gamma(z)$, where $\Gamma$ denotes the gamma function (Gutknecht, 1986):

$$
\psi_{p}(z)=\frac{(-1)^{p+1}}{p !} z^{p+1} \psi^{(p)}(z+1) .
$$

In order to calculate $\rho_{m}$ we have to evaluate $\psi_{4 d-1}$ in $z_{k}=k / N, k=0, \ldots, N-1$, i.e., $\psi^{(4 d-1)}$ at the values $z_{k}+1, k=0, \ldots, N-1$; these all lie in the interval [1,2) which does not contain the pole of $\psi^{(4 d-1)}$. Following Luke (1978, vol 1, p 2; vol 2, p 301), we can write

$$
\psi^{(p)}(x+1)=\psi^{(p)}(x+3)+(-1)^{p+1} p !\left[(x+1)^{-p-1}+(x+2)^{-p-1}\right],
$$

for $x \in[0,1]$. This formula allows us to use Luke's expansions for $\psi^{(p)}$ in the interval $[3,4]$ in terms of C̆ebyšev polynomials. Luke lists the coefficients of these Čbyšev polynomials for $p=0, \ldots, 6$. We evaluate $\psi_{p}$ at the negative values $-z_{k}, k=1, \ldots$, $N-1$, by using the relation $\psi_{p}(-z)=\sigma_{p}(z)-1-\psi_{p}(z)$, where $\sigma_{p}$ denotes the sigma function defined by

$$
\sigma_{p}(z):=\sum_{j=-\infty}^{+\infty}\left(\frac{z}{j+z}\right)^{p+1}= \begin{cases}\pi z \cot \pi z, & \text { for } p=0, \\ \operatorname{SINC}^{-(p+1)}(\pi z) q_{p-1}(\cos \pi z), & \text { for } p \in \mathbb{N} \backslash\{0\},\end{cases}
$$

and $q_{p-1}$ is the polynomial of degree $p-1$ defined recursively by

$$
q_{0}(t)=1, \quad q_{\ell}(t)=t q_{\ell-1}(t)+\frac{1-t^{2}}{\ell+1} q_{\ell-1}^{\prime}(t), \quad \ell=1,2, \ldots
$$

For the linear spline interpolant $(d=1)$ we get

$$
\rho_{m}=\tau_{m}^{2}\left[-\sigma_{3}\left(z_{m}\right)+1+\psi_{3}\left(z_{m}\right)+1+\psi_{3}\left(z_{m}\right)\right]=\tau_{m}^{2}\left[2-\sigma_{3}\left(z_{m}\right)+2 \psi_{3}\left(z_{m}\right)\right],
$$

whereas for the cubic spline $(d=2)$

$$
\rho_{m}=\tau_{m}^{2}\left[2-\sigma_{7}\left(z_{m}\right)+2 \psi_{7}\left(z_{m}\right)\right] .
$$

(b) Spline interpolants of odd order $2 d+1(d \geqslant 0)$.

Here

$$
\tau_{m+\ell N}=(-1)^{\ell}\left(\frac{m}{m+\ell N}\right)^{2 d+1} \tau_{m}
$$

and thus

$$
\rho_{m}=\tau_{m}^{2} \sum_{\ell=-\infty}^{+\infty} \operatorname{sign}(m+\ell N)\left(\frac{m}{m+\ell N}\right)^{4 d+2}=\tau_{m}\left(2-\sigma_{4 d+1}\left(z_{m}\right)+2 \psi_{4 d+1}\left(z_{m}\right)\right) .
$$


For the piecewise constant spline interpolant $(d=0)$ this yields

$$
\rho_{m}=\tau_{m}^{2}\left(2-\sigma_{1}\left(z_{m}\right)+2 \psi_{1}\left(z_{m}\right)\right) .
$$

Since the evaluation of each of the $\sigma_{p}$ and $\psi_{p}$ necessitates $\mathcal{O}(1)$ flops, their evaluation at all $z_{m}$ requires $\mathcal{O}(N)$ flops, and so does the calculation of all $\rho_{m}$.

\subsection{Evaluation of the approximate solution}

Once $\alpha$ and $\widehat{c}_{1}(\widehat{y}), \ldots, \widehat{c}_{N-1}(\widehat{y})$ have been calculated by solving the linear system (3.11) (directly or by the iterative method (3.13)), one needs to evaluate the approximation $\widehat{x}$ to $x$. We have

$$
\widehat{x}(t)=\alpha+\mathrm{K} \widehat{y}(t)=\alpha-\mathrm{i} \sum_{n=-\infty}^{+\infty} \operatorname{sign}(n) \tau_{n} \widehat{c}_{n}(\widehat{y}) \mathrm{e}^{\mathrm{i} n t} .
$$

This can again be written as a finite sum by collecting the factors of each of the different $\widehat{c}_{n}$ :

$$
\widehat{x}(t)=\alpha-\mathrm{i} \sum_{n=0}^{N-1} \widehat{c}_{n}(\widehat{y}) \mathrm{e}^{\mathrm{i} n t}\left[\sum_{\ell=-\infty}^{+\infty} \operatorname{sign}(n+\ell N) \tau_{n+\ell N} \mathrm{e}^{\mathrm{i} \ell N t}\right] .
$$

Let

$$
\delta_{n}(t):=\sum_{\ell=-\infty}^{+\infty} \operatorname{sign}(n+\ell N) \tau_{n+\ell N} \mathrm{e}^{\mathrm{i} \ell N t}
$$

If property (3.2) holds, then $\delta_{0}(t)=0$.

If we consider splines of even order $2 d(d \geqslant 1)$, then for $n \neq 0$

$$
\delta_{n}(t)=\tau_{n}\left[-\sum_{\ell=1}^{+\infty}\left(\frac{z_{n}}{-\ell+z_{n}}\right)^{2 d} \mathrm{e}^{-\mathrm{i} \ell N t}+1+\sum_{\ell=1}^{+\infty}\left(\frac{z_{n}}{\ell+z_{n}}\right)^{2 d} \mathrm{e}^{\mathrm{i} \ell N t}\right],
$$

where $z_{n}:=n / N, n=1, \ldots, N-1$. This can be expressed in terms of the generalized zeta-function, which figures among the predefined special functions in some programming languages (e.g. Mathematica, where it is known under the name LerchPhi), and which is defined by

$$
\zeta(s, a, z):=\sum_{k=0}^{\infty} \frac{z^{k}}{(k+a)^{s}}
$$

for any complex number $s$ with $\operatorname{Re}(s)>0$ if $|z| \leqslant 1, z \neq 1$, and for $s$ with $\operatorname{Re}(s)>1$ if $z=1, a \in \mathbb{R}$ (see, e.g., Hansen, 1975, p 142). From this definition we see that $\delta_{n}(t)$ can be written as

$$
\delta_{n}(t)=\tau_{n}\left[1+z_{n}^{2 d}\left(\zeta\left(2 d, z_{n}, \mathrm{e}^{\mathrm{i} N t}\right)-\zeta\left(2 d,-z_{n}, \mathrm{e}^{-\mathrm{i} N t}\right)\right)\right] .
$$

At the interpolation points $t_{k}=k 2 \pi / N$ this yields

$$
\delta_{n}\left(t_{k}\right)=\sum_{\ell=-\infty}^{+\infty} \operatorname{sign}(n+\ell N) \tau_{n+\ell N}=\tau_{n}\left[1+z_{n}^{2 d}\left(\zeta\left(2 d, z_{n}, 1\right)-\zeta\left(2 d,-z_{n}, 1\right)\right)\right] .
$$


Using the definition of $\psi_{p}(z)$ we see that $z_{n}^{2 d} \zeta\left(2 d, z_{n}, 1\right)=\psi_{2 d-1}\left(z_{n}\right)$ and so $\delta_{n}\left(t_{k}\right)$ can be expressed as

$$
\delta_{n}\left(t_{k}\right)=\tau_{n}\left[-\psi_{2 d-1}\left(-z_{n}\right)+1+\psi_{2 d-1}\left(z_{n}\right)\right]=\tau_{n}\left[2-\sigma_{2 d-1}\left(z_{n}\right)+2 \psi_{2 d-1}\left(z_{n}\right)\right],
$$

where $\psi_{2 d-1}\left(z_{n}\right)$ is computed through the polygamma function $\psi^{(2 d-1)}$, as in the previous section.

For odd orders $2 d+1(d \geqslant 0)$,

$$
\begin{aligned}
\delta_{n}(t) & =\sum_{\ell=-\infty}^{+\infty} \operatorname{sign}(n+\ell N)(-1)^{\ell}\left(\frac{n}{\ell N+n}\right)^{2 d+1} \tau_{n} \mathrm{e}^{\mathrm{i} \ell N t} \\
& =\tau_{n}\left[\sum_{\ell=1}^{+\infty}(-1)^{\ell}\left(\frac{-z_{n}}{\ell-z_{n}}\right)^{2 d+1} \mathrm{e}^{\mathrm{i} \ell N t}+1+\sum_{\ell=1}^{+\infty}(-1)^{\ell}\left(\frac{z_{n}}{\ell+z_{n}}\right)^{2 d+1} \mathrm{e}^{\mathrm{i} \ell N t}\right]
\end{aligned}
$$

and in terms of the zeta-function

$$
\delta_{n}(t)=\tau_{n}\left[-1+z_{n}^{2 d+1}\left(\zeta\left(2 d+1,-z_{n},-\mathrm{e}^{-\mathrm{i} N t}\right)+\zeta\left(2 d+1, z_{n},-\mathrm{e}^{\mathrm{i} N t}\right)\right)\right] .
$$

At the interpolation points, $\delta_{n}$ can again be expressed in terms of the polygamma function. For this purpose, let us define

$$
\widetilde{\psi}_{p}(z):=\sum_{\ell=1}^{+\infty}(-1)^{\ell}\left(\frac{z}{z+\ell}\right)^{p+1} .
$$

Then we have

$$
\delta_{n}\left(t_{k}\right)=\tau_{n}\left[-\widetilde{\psi}_{2 d}\left(-z_{n}\right)+1+\widetilde{\psi}_{2 d}\left(z_{n}\right)\right]
$$

and making use of the relation $\widetilde{\psi}_{p}(-z)=\widetilde{\sigma}_{p}(z)-1-\widetilde{\psi}_{p}(z)$, where $\widetilde{\sigma}_{p}$ is defined by

$$
\widetilde{\sigma}_{p}(z):=\sum_{j=-\infty}^{+\infty}(-1)^{j}\left(\frac{z}{j+z}\right)^{p+1}=\operatorname{SINC}^{-(p+1)}(\pi z) \widetilde{q}_{p}(\cos \pi z)
$$

and $\widetilde{q}_{\ell}$ is a polynomial defined recursively by

$$
\widetilde{q}_{0}(t):=1, \quad \tilde{q}_{\ell}(t):=t \widetilde{q}_{\ell-1}(t)+\frac{1-t^{2}}{\ell} \widetilde{q}_{\ell-1}^{\prime}(t), \quad \ell=1,2, \ldots,
$$

$\delta_{n}\left(t_{k}\right)$ becomes

$$
\delta_{n}\left(t_{k}\right)=\tau_{n}\left[2-\widetilde{\sigma}_{2 d}\left(z_{n}\right)+2 \widetilde{\psi}_{2 d}\left(z_{n}\right)\right] .
$$

If $t$ is one of the midpoints between the interpolation points, i.e., $t=t_{k}+\pi / N$ for some $k \in\{0, \ldots, N-1\}$, then we have to evaluate

$$
\delta_{n}\left(t_{k}+\pi / N\right)=\sum_{\ell=-\infty}^{+\infty}(-1)^{\ell} \operatorname{sign}(n+\ell N) \tau_{n+\ell N} .
$$


In the case of spline interpolation of even order $2 d(d \geqslant 1)$ we get by similar calculations as for $\delta_{n}\left(t_{k}\right)$

$$
\delta_{n}\left(t_{k}+\pi / N\right)=\tau_{n}\left[2-\widetilde{\sigma}_{2 d-1}\left(z_{n}\right)+2 \widetilde{\psi}_{2 d-1}\left(z_{n}\right)\right]
$$

and for the splines of odd order $2 d+1(d \geqslant 0)$

$$
\delta_{n}\left(t_{k}+\pi / N\right)=\tau_{n}\left[2-\sigma_{2 d}\left(z_{n}\right)+2 \psi_{2 d}\left(z_{n}\right)\right] .
$$

The functions $\widetilde{\psi}_{p}$ appearing in (3.14) and (3.15) are absent from most libraries, in contrast to the polygamma function $\psi^{(p)}(z)$, which is available as an intrinsic function in many programming languages (e.g., Mathematica, Maple, MATLAB, etc). In order to express the series $\widetilde{\psi}_{p}\left(z_{n}\right)$ in terms of the functions $\psi^{(p)}$ we define the series

$$
\beta(z):=\sum_{\ell=0}^{+\infty} \frac{(-1)^{\ell}}{(z+\ell)} .
$$

It can easily be verified that

$$
\beta(z)=\frac{1}{2}\left[\psi\left(\frac{z+1}{2}\right)-\psi\left(\frac{z}{2}\right)\right] .
$$

With the above notation and formulas, we have

$$
\widetilde{\psi}_{p}(z)=\frac{z^{p+1}(-1)^{p}}{p !} \beta^{(p)}(z)-1=\frac{z^{p+1}(-1)^{p}}{2^{p+1} p !}\left[\psi^{(p)}\left(\frac{z+1}{2}\right)-\psi^{(p)}\left(\frac{z}{2}\right)\right]-1 .
$$

To avoid the pole at $z=0$ we finally transform the last equation using $\psi^{(p)}(z+1)=$ $\psi^{(p)}(z)+(-1)^{p} p ! z^{-p-1}$ into

$$
\widetilde{\psi}_{p}(z)=\frac{z^{p+1}(-1)^{p}}{p ! 2^{p+1}}\left[\psi^{(p)}\left(\frac{z+1}{2}+1\right)-\psi^{(p)}\left(\frac{z}{2}+1\right)\right]-\left(\frac{z}{z+1}\right)^{p+1} .
$$

Thus, after the $\widehat{c}_{n}(\widehat{y})$ have been determined through the linear system (3.11), $\mathcal{O}(N)$ flops are needed to evaluate $\widehat{x}$ at the $N$ interpolation points and/or intermediate points.

\section{Rates of convergence of the approximate solution}

As noted in the literature (see, e.g., Atkinson, 1997), one advantage of degenerate-kernel methods is the simplicity of their analysis. Consider the approximate equation $\widehat{x}+\widehat{\mathrm{T}} \widehat{x}=\widehat{g}$ with $\widehat{T}$ and $\widehat{g}$ defined in (3.6) and (3.7). We can use a convergence theorem of Schleiff for such operator equations of the second kind in $\mathrm{L}_{2}(I)$ (see Schleiff, 1968, p 480, for the proof) or the theorems on operator approximations in Kress (1989, Chapter 10).

THEOREM 4.1 Suppose that $(\mathrm{I}+\mathrm{T})^{-1}$ exists and that the following two conditions are satisfied:

$$
\|\mathrm{T}-\widehat{\mathrm{T}}\| \rightarrow 0 \quad \text { in the } \mathrm{L}_{2} \text {-norm }
$$

and

$$
\|g-\widehat{g}\| \rightarrow 0 \quad \text { in the } \mathrm{L}_{2} \text {-norm. }
$$


Then for some $N_{0} \in \mathbb{N}$ the approximate equation $\widehat{x}+\widehat{T} \widehat{x}=\widehat{g}$ is uniquely solvable in $\mathrm{L}_{2}(I)$ for $N \geqslant N_{0}$ and the solution $\widehat{x}$ converges towards the exact solution $x$. Moreover, the following error bound holds:

$$
\|x-\widehat{x}\| \leqslant\left\|(\mathrm{I}+\mathrm{T})^{-1}\right\|\left[2\|\mathrm{~T}-\widehat{\mathrm{T}}\|\left\|(\mathrm{I}+\mathrm{T})^{-1}\right\|\|\widehat{g}\|+\|g-\widehat{g}\|\right] .
$$

We have seen in Section 2 that, for every piecewise $\mathrm{C}^{2}, \mathrm{C}^{1}$-Jordan curve, and for every piecewise regular $\mathrm{C}^{2}$-Jordan curve without cusps and with logarithmic capacity different from $1, \mathrm{I}+\mathrm{S}^{-1} \mathrm{R}$ has a bounded inverse so that the first condition is satisfied. To verify (4.1) and (4.2) let us bound $\|\widehat{T} x-\mathrm{T} x\|_{\mathrm{L}_{2}}$ for $x \in \mathrm{L}_{2}(I)$ and $\|g-\widehat{g}\|_{\mathrm{L}_{2}}$. We have

$$
\begin{aligned}
\|\widehat{\mathrm{T}} x-\mathrm{T} x\|_{\mathrm{L}_{2}} & =\left\|-2(\operatorname{Int} \mathrm{K} \widehat{\mathrm{D} r}) x+c_{0}(\widehat{\mathrm{R}} x)+2(\operatorname{Int} \mathrm{KD} r) x-c_{0}(\mathrm{R} x)\right\| \\
& \leqslant(\|\mathrm{R}-\widehat{\mathrm{R}}\|+2\|\mathrm{~K}\|\|\mathrm{DR}-\widehat{\mathrm{DR}}\|)\|x\|
\end{aligned}
$$

and

$$
\|g-\widehat{g}\|_{\mathrm{L}_{2}}=\left\|-2 \mathrm{~K} \widehat{\mathrm{D} f}+c_{0}(\widehat{f})+2 \mathrm{KD} f-c_{0}(f)\right\| \leqslant 2\|\mathrm{~K}\|\|\mathrm{D} f-\widehat{\mathrm{D} f}\|+\|\widehat{f}-f\| .
$$

We thus have the following theorem.

THEOREM 4.2 The convergence of $\widehat{x}$ toward $x$ as $N \rightarrow \infty$ is guaranteed if $\| \widehat{\mathrm{D} r}-$ $\mathrm{D} r \|_{\mathrm{L}_{2}} \rightarrow 0$ and if $\|\widehat{\mathrm{D} f}-D f\|_{\mathrm{L}_{2}} \rightarrow 0$ as $N \rightarrow \infty$. Moreover, if $\Gamma$ is such that $\| \widehat{\mathrm{D} r}-$ $\mathrm{D} r \|_{\mathrm{L}_{2}} \leqslant \mathcal{O}\left(h^{k}\right)$ and $\|\widehat{\mathrm{D} f}-D f\|_{\mathrm{L}_{2}} \leqslant \mathcal{O}\left(h^{\ell}\right)$ then

$$
\|\widehat{x}-x\|_{\mathrm{L}_{2}} \leqslant \mathcal{O}\left(h^{\min \{k, \ell\}}\right) .
$$

In the case of trigonometric interpolation, we only need the Riemann integrability of $r, \mathrm{D} r$, $f$ and $\mathrm{D} f$ for convergence (see Berrut, 1986). Recalling the properties of $r$ and $\mathrm{D} r$ given in Section 2 we see that this holds for every piecewise $\mathrm{C}^{2}, \mathrm{C}^{1}$-Jordan curve $\Gamma$. Then, for every right-hand side function $f$ such that $f$ and $\mathrm{D} f$ are Riemann integrable, the approximate equation (3.8) has a unique solution $\widehat{x}$, and $\widehat{x} \rightarrow x$ as $N \rightarrow \infty$. From the convergence results for one- and two-dimensional trigonometric interpolating polynomials (see Canuto et al., 1988) we conclude that $\|x-\widehat{x}\|_{\mathrm{L}_{2}}=\mathcal{O}\left(h^{m}\right)$, where $h:=2 \pi / N$, if $\mathrm{D} f \in \mathrm{H}^{m}(I)$ and $\mathrm{D} r \in \mathrm{H}^{m}\left(I^{2}\right)$ for $m>1$. On the other hand, if $\Gamma$ is analytic, then exponential rates of convergence can be expected if $f$ is analytic (Berrut, 1986).

In the case of spline interpolants the rates of convergence depend, as for trigonometric interpolation, on the smoothness of the curve $\Gamma$ and of the inhomogeneity $f$. But they are also restricted by the order of the splines. Thus, while the trigonometric polynomials automatically fit to the order of differentiability, the latter order has to be known to choose accordingly the spline degree. (On the other hand, the spline interpolant oscillates less between the interpolation points.) Using the approximation power of spline and tensorproduct spline interpolants (Schumaker, 1981), we see that the maximal order $\mathcal{O}\left(h^{k}\right)$ is reached if the kernel $\mathrm{D} r$ belongs to the tensor-product Sobolev space $\mathrm{H}^{(k, k)}\left(I^{2}\right)$, and if $\mathrm{D} f$ belongs to $\mathrm{H}^{k}(I)$. If $\mathrm{D} r \in \mathrm{H}^{(m, m)}\left(I^{2}\right)$ and $\mathrm{D} f \in \mathrm{H}^{p}(I)$ with $m<k$ or $p<k, k \geqslant 1$, then the rate of convergence is $\mathcal{O}\left(h^{\min \{m, p\}}\right)$.

In the literature (e.g. Schumaker, 1981) we have merely found convergence results in tensor Sobolev spaces $\mathrm{H}^{(p, q)}\left(I^{2}\right)$ for integer $p$ and $q$. However, numerical experiments demonstrate that similar results should hold for real-valued exponents also. 
In the next section we report on experiments for Symm's equation with piecewise analytic $\mathrm{C}^{1}$ and $\mathrm{C}^{2}$-curves. Analysing the Fourier series, we see that the corresponding parametric representations $z(t)$ belong to $\mathrm{H}^{2 \cdot 5-\varepsilon}(I)$ and $\mathrm{H}^{3 \cdot 5-\varepsilon}(I), \varepsilon>0$, and the righthand side functions $g(t)$ belong to $\mathrm{H}^{1 \cdot 5-\varepsilon}(I)$ and $\mathrm{H}^{2 \cdot 5-\varepsilon}(I)$, respectively. From the known behaviour of the solution $x$ at the corners (Lehman, 1957, 1959), it is easy to see (Hoidn, 1983 ) that, for piecewise analytic $\mathrm{C}^{1}$-curves, the exact solution $x$ belongs to $\mathrm{H}^{p}(I)$ for all $p<1 \cdot 5$. Since T $x$ belongs to this same Sobolev space, it follows that the kernel function $\mathrm{D} r$ is in the tensor Sobolev space $\mathrm{H}^{(p, p+1)}\left(I^{2}\right) \supset \mathrm{H}^{(p, p)}\left(I^{2}\right)$. Hence the convergence of $\widehat{x}$ towards $x$ in the $\mathrm{L}_{2}$-norm can be expected to be $\mathcal{O}\left(h^{\min \{1 \cdot 5-\varepsilon, k\}}\right), \varepsilon>0$, where $k$ is the order of the spline interpolant. In the same way we deduce $\mathcal{O}\left(h^{\min \{2 \cdot 5-\varepsilon, k\}}\right)$ convergence rates for $\mathrm{C}^{2}$-curves.

\section{Some numerical results}

\section{EXAMPLE 1 Conformal maps}

Let $\Gamma: z(t)$ be a Jordan curve with $0 \in G:=\operatorname{int}(\Gamma)$ and with logarithmic capacity different from one. The function $h$ which conformally maps $G$ onto the unit circle with $h(0)=0, \arg \left(h^{\prime}(0)\right)=0$ is then given by

$$
h(z)=z \mathrm{e}^{U(z)+\mathrm{i} V(z)},
$$

where $U$ is the solution of the Dirichlet problem

$$
\begin{gathered}
\Delta U=0 \text { in } G, \\
U(z)=-\log |z| \text { on } \Gamma
\end{gathered}
$$

and $V$ is the function conjugate harmonic to $U$ with $V(0)=0$. The boundary integral equation (2.1) then becomes

$$
\frac{1}{2 \pi} \int_{0}^{2 \pi} \log |z(t)-z(s)| x(s) \mathrm{d} s=\log |z(t)|,
$$

where the charge density $x$ is the derivative of the boundary correspondence function $\theta$. Equation (5.3) is called Symm's equation. In a first test, we have applied the method of Section 3 to an analytic curve, namely the ellipse with semi-axes $\beta(1+\eta)$ and $\beta(1-\eta)$, whose parametric representation is given by

$$
z(t)=\beta\left(\mathrm{e}^{\mathrm{i} t}+\eta \mathrm{e}^{-\mathrm{i} t}\right) .
$$

As a second example we have constructed a piecewise analytic $\mathrm{C}^{1}$-curve by combining one half of an ellipse with one half of a circle, and $\mathrm{C}^{2}$-curves by interpolating values of known analytic curves, such as the ellipses (5.4), with periodic cubic splines. Finally we have mapped the square of side length $\pi / 2$ with the parametric representation

$$
z(t)= \begin{cases}\frac{\pi}{4}+\mathrm{i} t, & \text { for } 0 \leqslant t \leqslant \frac{\pi}{4}, \\ \frac{\pi}{2}-t+\mathrm{i} \frac{\pi}{4}, & \text { for } \frac{\pi}{4} \leqslant t \leqslant \frac{\pi}{2},\end{cases}
$$


periodically repeated on $[\pi / 2,2 \pi]$. The derivatives at the corners have been computed as the arithmetic means of their left and right derivatives, in accordance with the behaviour of Fourier series of piecewise smooth functions at jumps.

The numerical results for the four curves described above and for spline approximation operators of different orders are shown in Tables 1 to 4 . We give the experimental $\mathrm{L}_{2}$ errors for $N$ points in $\widehat{\theta}_{N}^{\prime}:=\widehat{\theta}^{\prime}$ or $\widehat{\theta}_{N}:=\widehat{\theta}$ and the corresponding convergence rates for increasing values of $N$. Analytical expressions of $\theta^{\prime}$ and $\theta$ are known for the ellipse (see Henrici, 1979, p 494) so that we can compute the experimental convergence rate as

$$
\text { ecr }=\log _{2}\left(\sum_{k=0}^{2047}\left|\theta^{\prime}\left(\xi_{k}\right)-\widehat{\theta}_{N}^{\prime}\left(\xi_{k}\right)\right|^{2} / \sum_{k=0}^{2047}\left|\theta^{\prime}\left(\xi_{k}\right)-\widehat{\theta}_{2 N}^{\prime}\left(\xi_{k}\right)\right|^{2}\right)^{1 / 2}
$$

where $\xi_{k}:=k 2 \pi / 2048, k=0, \ldots, 2047$. Whenever the exact solution is not known we replace $\theta^{\prime}$ (resp. $\theta$ ) by $\widehat{\theta}_{2048}^{\prime}$ (resp. $\widehat{\theta}_{2048}$ ) in the computation of these experimental $\mathrm{L}_{2}$-errors and convergence rates.

Table 1 displays the experimental convergence rates when $\Gamma$ is an ellipse and for splines of order 1,2 and 4. These rates can be compared with the theoretical rates 1,2 and 4 .

In the case of the $\mathrm{C}^{2}$-curve, we have seen in Section 4 that the expected convergence rates are $\mathcal{O}\left(h^{\min \{2 \cdot 5-\varepsilon, k\}}\right)$, where $k$ is the order of the spline interpolant. These rates are confirmed by the results in Table 2; the value of ecr in the case of cubic spline interpolation is approximatively $2 \cdot 5$ and about 1 , respectively 2 , for splines of order 1 , respectively 2 . In Table 3 we see that for the $\mathrm{C}^{1}$-curve the experimental convergence rates for splines of order 1,2 and 4 are approximately $1,1.5$ and 1.5, which corresponds to the convergence rates of $\mathcal{O}\left(h^{\min \{1 \cdot 5-\varepsilon, k\}}\right)$ found in Section 4.

The exact boundary correspondence function $\theta$ for the square can be found in Gaier (1964), for example. In Table 4 we observe experimental convergence rates of approximately $2,2.5$ and 2.5 for $\widehat{\theta}$ with piecewise constant, linear and cubic spline interpolants. For $\widehat{\theta}^{\prime}$ this corresponds to an $\mathcal{O}(h)$-convergence for piecewise constant splines and an $\mathcal{O}\left(h^{1 \cdot 5}\right)$-convergence for linear and cubic splines. Since the kernel function merely belongs to $\mathrm{H}^{-\varepsilon}\left(I^{2}\right)$, convergence is not ensured by Theorem 4.2.

EXAMPLE 2 Interior Dirichlet problem for the potential $U(z)=\operatorname{Re}\left(z^{2}\right)$. In the case of the ellipses (5.4) the boundary condition (1.3) is

$$
f(t)=U(z(t))=\beta^{2}\left(\left(1+\eta^{2}\right) \cos 2 t+2 \eta\right) .
$$

Let $\widehat{x}(t)$ be the solution of equation (2.1) for these Dirichlet data $f$. Then the approximate potential $\widehat{U}$ is given by

$$
\widehat{U}(z):=\frac{1}{2 \pi} \int_{0}^{2 \pi} \log |z-z(s)| \widehat{x}(s) \mathrm{d} s .
$$

Table 5 shows some error and convergence results for the approximate density function $\widehat{x}(t)$ for the ellipse with $\eta=1 / 3$ in the case of spline interpolation. For cubic splines we observe the expected $\mathcal{O}\left(h^{4}\right)$ convergence. 
TABle 1

$L_{2}$-errors in $\widehat{\theta}^{\prime}$ and experimental convergence rate for the ellipse with $\eta=1 / 3$ and for splines of order $k$.

\begin{tabular}{|c|c|c|c|c|c|c|}
\hline \multirow[b]{2}{*}{$N$} & \multicolumn{2}{|l|}{$k=1$} & \multicolumn{2}{|l|}{$k=2$} & \multicolumn{2}{|l|}{$k=4$} \\
\hline & $\mathrm{L}_{2}$-errors & ecr & $\mathrm{L}_{2}$-errors & ecr & $\mathrm{L}_{2}$-errors & ecr \\
\hline 32 & $2 \cdot 2852 \times 10^{-1}$ & & $3.6961 \times 10^{-2}$ & & $4.3233 \times 10^{-4}$ & \\
\hline 64 & $1.0814 \times 10^{-1}$ & $1 \cdot 079$ & $1 \cdot 1738 \times 10^{-2}$ & $1 \cdot 654$ & $4.7428 \times 10^{-5}$ & $3 \cdot 188$ \\
\hline 128 & $5 \cdot 2446 \times 10^{-2}$ & $1 \cdot 044$ & $3 \cdot 1999 \times 10^{-3}$ & $1 \cdot 875$ & $3 \cdot 8082 \times 10^{-6}$ & $3 \cdot 638$ \\
\hline 256 & $2.5807 \times 10^{-2}$ & $1 \cdot 023$ & $8.2724 \times 10^{-4}$ & $1 \cdot 951$ & $2.6866 \times 10^{-7}$ & $3 \cdot 825$ \\
\hline 512 & $1.2798 \times 10^{-2}$ & $1 \cdot 011$ & $2.0975 \times 10^{-4}$ & 1.979 & $1.7825 \times 10^{-8}$ & $3 \cdot 913$ \\
\hline 1024 & $6.3727 \times 10^{-3}$ & $1 \cdot 006$ & $5 \cdot 2776 \times 10^{-5}$ & $1 \cdot 990$ & $1.1475 \times 10^{-9}$ & 3.987 \\
\hline
\end{tabular}

TABLE 2

$L_{2}$-errors in $\widehat{\theta}^{\prime}$ and experimental convergence rates for the $C^{2}$-curve approximating an ellipse with $\eta=1 / 3$ and for splines of order $k$.

\begin{tabular}{|c|c|c|c|c|c|c|}
\hline \multirow[b]{2}{*}{$N$} & \multicolumn{2}{|l|}{$k=1$} & \multicolumn{2}{|l|}{$k=2$} & \multicolumn{2}{|l|}{$k=4$} \\
\hline & $\mathrm{L}_{2}$-errors & ecr & $\mathrm{L}_{2}$-errors & ecr & $\mathrm{L}_{2}$-errors & ecr \\
\hline 16 & $1 \cdot 2814 \times 10^{-1}$ & & $8.9517 \times 10^{-1}$ & & $4.3946 \times 10^{-2}$ & \\
\hline 32 & $5 \cdot 4901 \times 10^{-2}$ & 1.222 & $1.9477 \times 10^{-2}$ & $5 \cdot 522$ & $1.0534 \times 10^{-3}$ & $5 \cdot 299$ \\
\hline 64 & $2 \cdot 5722 \times 10^{-2}$ & $1 \cdot 093$ & $4.9434 \times 10^{-3}$ & 1.978 & $6 \cdot 8179 \times 10^{-5}$ & $4 \cdot 032$ \\
\hline 128 & $1 \cdot 2411 \times 10^{-2}$ & $1 \cdot 051$ & $1.2399 \times 10^{-3}$ & 1.995 & $9 \cdot 7672 \times 10^{-6}$ & $2 \cdot 803$ \\
\hline 256 & $6.0916 \times 10^{-3}$ & $1 \cdot 026$ & $3.0928 \times 10^{-4}$ & $2 \cdot 003$ & $1.6308 \times 10^{-6}$ & $2 \cdot 582$ \\
\hline 512 & $3 \cdot 0171 \times 10^{-3}$ & $1 \cdot 014$ & $7 \cdot 5794 \times 10^{-5}$ & $2 \cdot 028$ & $2 \cdot 8251 \times 10^{-7}$ & $2 \cdot 529$ \\
\hline
\end{tabular}

TABLE 3

$L_{2}$-errors in $\widehat{\theta}^{\prime}$ and experimental convergence rates for the $C^{1}$-curve and for splines of order $k$.

\begin{tabular}{|c|c|c|c|c|c|c|}
\hline \multirow[b]{2}{*}{$N$} & \multicolumn{2}{|l|}{$k=1$} & \multicolumn{2}{|l|}{$k=2$} & \multicolumn{2}{|l|}{$k=4$} \\
\hline & $\mathrm{L}_{2}$-errors & ecr & $\mathrm{L}_{2}$-errors & ecr & $\mathrm{L}_{2}$-errors & ecr \\
\hline 16 & $2.9540 \times 10^{-2}$ & & $1.2309 \times 10^{-1}$ & & $1.5712 \times 10^{-2}$ & \\
\hline 32 & $1.3365 \times 10^{-2}$ & $1 \cdot 106$ & $2.9549 \times 10^{-3}$ & $5 \cdot 380$ & $4.4916 \times 10^{-3}$ & 1.806 \\
\hline 64 & $6 \cdot 2345 \times 10^{-3}$ & $1 \cdot 100$ & $9 \cdot 3719 \times 10^{-4}$ & $1 \cdot 656$ & $1.4970 \times 10^{-3}$ & $1 \cdot 585$ \\
\hline 128 & $2.9781 \times 10^{-3}$ & $1 \cdot 065$ & $3.0440 \times 10^{-4}$ & $1 \cdot 622$ & $5 \cdot 1691 \times 10^{-4}$ & $1 \cdot 534$ \\
\hline 256 & $1.4499 \times 10^{-3}$ & $1 \cdot 038$ & $1.0258 \times 10^{-4}$ & $1 \cdot 583$ & $1.8061 \times 10^{-4}$ & $1 \cdot 517$ \\
\hline 512 & $7 \cdot 1419 \times 10^{-4}$ & $1 \cdot 021$ & $6.3452 \times 10^{-5}$ & $1 \cdot 509$ & $6 \cdot 3452 \times 10^{-5}$ & $1 \cdot 509$ \\
\hline
\end{tabular}


TABLE 4

$L_{2}$-errors in $\widehat{\theta}$ and experimental convergence rates for the square and for splines of order $k$.

\begin{tabular}{|c|c|c|c|c|c|c|}
\hline \multirow[b]{2}{*}{$N$} & \multicolumn{2}{|l|}{$k=1$} & \multicolumn{2}{|l|}{$k=2$} & \multicolumn{2}{|l|}{$k=4$} \\
\hline & $\mathrm{L}_{2}$-errors & ecr & $\mathrm{L}_{2}$-errors & ecr & $\mathrm{L}_{2}$-errors & ecr \\
\hline 32 & $1.0162 \times 10^{-2}$ & & $1.6872 \times 10^{-2}$ & & $5 \cdot 7658 \times 10^{-3}$ & \\
\hline 64 & $3 \cdot 1095 \times 10^{-3}$ & $1 \cdot 708$ & $3 \cdot 3695 \times 10^{-3}$ & $2 \cdot 324$ & $9 \cdot 2885 \times 10^{-4}$ & $2 \cdot 634$ \\
\hline 128 & $8 \cdot 3204 \times 10^{-4}$ & 1.901 & $6.0859 \times 10^{-4}$ & $2 \cdot 469$ & $1.5849 \times 10^{-4}$ & $2 \cdot 551$ \\
\hline 256 & $2 \cdot 1249 \times 10^{-4}$ & 1.969 & $1.0901 \times 10^{-4}$ & $2 \cdot 481$ & $2 \cdot 7671 \times 10^{-5}$ & $2 \cdot 518$ \\
\hline 512 & $5 \cdot 3290 \times 10^{-5}$ & 1.995 & $1.9005 \times 10^{-5}$ & $2 \cdot 520$ & $4 \cdot 8645 \times 10^{-6}$ & $2 \cdot 508$ \\
\hline
\end{tabular}

TABLE 5

$L_{2}$-errors in $\widehat{x}$ and experimental convergence rates in Example 2 for the ellipse with $\eta=1 / 3$ and for splines of order $k$.

\begin{tabular}{|c|c|c|c|c|c|c|}
\hline \multirow[b]{2}{*}{$N$} & \multicolumn{2}{|l|}{$k=1$} & \multicolumn{2}{|l|}{$k=2$} & \multicolumn{2}{|l|}{$k=4$} \\
\hline & $\mathrm{L}_{2}$-errors & ecr & $\mathrm{L}_{2}$-errors & ecr & $\mathrm{L}_{2}$-errors & ecr \\
\hline 32 & $2 \cdot 2852 \times 10^{-1}$ & & $3.6961 \times 10^{-2}$ & & $4.3233 \times 10^{-4}$ & \\
\hline 64 & $1.0814 \times 10^{-1}$ & $1 \cdot 079$ & $1 \cdot 1738 \times 10^{-2}$ & $1 \cdot 654$ & $4.7428 \times 10^{-5}$ & $3 \cdot 188$ \\
\hline 128 & $5 \cdot 2446 \times 10^{-2}$ & $1 \cdot 044$ & $3 \cdot 1999 \times 10^{-3}$ & $1 \cdot 875$ & $3 \cdot 8082 \times 10^{-6}$ & $3 \cdot 638$ \\
\hline 256 & $2.5807 \times 10^{-2}$ & $1 \cdot 023$ & $8.2724 \times 10^{-4}$ & $1 \cdot 951$ & $2.6866 \times 10^{-7}$ & $3 \cdot 825$ \\
\hline 512 & $1.2798 \times 10^{-2}$ & $1 \cdot 011$ & $2.0975 \times 10^{-4}$ & 1.979 & $1.7825 \times 10^{-8}$ & $3 \cdot 913$ \\
\hline 1024 & $6.3727 \times 10^{-3}$ & 1.006 & $5.2776 \times 10^{-5}$ & 1.990 & $1 \cdot 1475 \times 10^{-9}$ & $3 \cdot 987$ \\
\hline
\end{tabular}

Finally we have used the $N$-point trapezoidal rule to evaluate $\widehat{U}(z)$ and we denote the corresponding values by $\widehat{U}^{(N)}(z)$. In Table 6 we list the absolute error $\left|U(z)-\widehat{U}^{(N)}(z)\right|$ of the potential at the points $z_{1}=(0 \cdot 0,0 \cdot 0), z_{2}=(2 \cdot 0,1 \cdot 0)$ and $z_{3}=(3 \cdot 8,0 \cdot 0)$, as well as the experimental convergence rate ecr $:=\log _{2}\left(\left|U(z)-\widehat{U}^{(N)}(z)\right| /\left|U(z)-\widehat{U}^{(2 N)}(z)\right|\right)$ at these points.

Further interesting examples of approximation operators which can be used with our method are operators which are only defined in Fourier spaces as linear and translation invariant smoothing operators like cosine-, Cesáro-, Lanczos- and spline-smoothing, see Berrut \& Reifenberg (1997).

The number of iterations required for numerical convergence is usually small, but depends on the shape of the curve $\Gamma$. Less than 10 iterations are needed for many curves and 40 iterations are usually sufficient. Only for curves with reentrant arcs like reflected ellipses and small parameters (Berrut, 1985) did we have to compute between 100 and 130 iterations for convergence to machine precision. 
TABLE 6

Absolute error in $\widehat{U}^{(N)}$ and experimental convergence rates at the points $z_{1}=(0 \cdot 0,0 \cdot 0), z_{2}=$ $(2 \cdot 0,1 \cdot 0)$ and $z_{3}=(3 \cdot 8,0 \cdot 0)$ for the ellipse with $\eta=1 / 3$ and for splines of order 4 .

\begin{tabular}{rlllllr}
\hline$N$ & $\left|U\left(z_{1}\right)-\widehat{U}^{(N)}\left(z_{1}\right)\right|$ & ecr & $\left|U\left(z_{2}\right)-\widehat{U}^{(N)}\left(z_{2}\right)\right|$ & ecr & $\left|U\left(z_{3}\right)-\widehat{U}^{(N)}\left(z_{3}\right)\right|$ & ecr \\
\hline 64 & $1.0734 \times 10^{-5}$ & & $4.6874 \times 10^{-6}$ & & $2 \cdot 0525 \times 10^{-2}$ & \\
128 & $6.2477 \times 10^{-7}$ & 4.103 & $2 \cdot 7767 \times 10^{-7}$ & 4.077 & $5.6430 \times 10^{-4}$ & $5 \cdot 643$ \\
256 & $3.7679 \times 10^{-8}$ & 4.051 & $1.6746 \times 10^{-8}$ & $4 \cdot 051$ & $2 \cdot 8209 \times 10^{-7}$ & $10 \cdot 507$ \\
512 & $2.3130 \times 10^{-9}$ & 4.026 & $1.0279 \times 10^{-9}$ & 4.026 & $3.8719 \times 10^{-9}$ & $6 \cdot 181$ \\
1024 & $1.4286 \times 10^{-10}$ & 4.017 & $6.3402 \times 10^{-11}$ & 4.019 & $2.3977 \times 10^{-10}$ & 4.015 \\
2048 & $8.8364 \times 10^{-12}$ & 4.015 & $3.2409 \times 10^{-12}$ & 4.290 & $1.4827 \times 10^{-11}$ & 4.013 \\
\hline
\end{tabular}

\section{Acknowledgements}

The idea for the work presented here originated in discussions with Martin Gutknecht on attenuation factors. The authors also wish to thank the referees for their comments which have improved the article's appearance.

\section{REFERENCES}

ARNOLD, D. N. 1983 A spline-trigonometric Galerkin method and an exponentially convergent boundary integral method. Math. Comput. 41, 383-397.

AtKinson, K. E. 1988 A discrete Galerkin method for first kind integral equations with a logarithmic kernel. J. Int. Equations Applic. 1, 343-363.

AtKinson, K. E. 1997 The Numerical Solution of Integral Equations of the Second Kind. Cambridge: Cambridge University Press.

BerRUT, J.-P. 1976 Numerische Lösung der Symmschen Integralgleichung durch FourierMethoden. Diplomarbeit, ETH Zürich.

BERRUT, J.-P. 1985 Integralgleichungen und Fourier-Methoden zur numerischen konformen Abbildung. PhD Thesis, ETH Zürich.

BERRUT, J.-P. 1986 A Fredholm integral equation of the second kind for conformal mapping. $J$. Comput. Appl. Math. 14, 99-110.

Berrut, J.-P. \& Reifenberg, M. 1997 Numerical solution of periodic Fredholm integral equations of the second kind by means of attenuation factors. J. Int. Equations Appl. 9, 1-20.

Canuto, C., Hussaini, M. Y., Quarteroni, A., \& ZAng, T. 1988 Spectral Methods in Fluid Dynamics. New York: Springer.

Chandler, G. A. \& SloAn, I. H. 1990 Spline qualocation methods for boundary integral equations. Numer. Math. 58, 537-567.

Elschner, J. \& GRAham, I. G. 1994 Quadrature methods for Symm's integral equation on polygons. Preprint No 94-21, School of Mathematical Sciences, University of Bath.

ELSCHNER, J. \& GRAHAM, I. G. 1995 An optimal order collocation method for first kind boundary integral equations on polygons. Numer. Math. 70, 1-31.

GAIER, D. 1964 Konstruktive Methoden der konformen Abbildung. Berlin: Springer.

GAIER, D. 1976 Integralgleichungen erster Art und konforme Abbildung. Math. Z. 147, 113-129.

GAUTSCHI, W. 1972 Attenuation factors in practical Fourier analysis. Numer. Math. 18, 373-400.

GrahAM, I. G. \& YAN, I. 1990 Boundary integral methods for Laplace's equation. Numerical Analysis 1989 (D. F. Griffiths and G. A. Watson, eds). Harlow: Longman, pp 122-136. 
GuTKNECHT, M. H. 1986 The evaluation of the conjugate function of a periodic spline on a uniform mesh. J. Comput. Appl. Math. 16, 181-201.

Gutknecht, M. H. 1987 Attenuation factors in multivariate Fourier analysis. Numer. Math. 51, 615-629.

HALMOS, P. R. \& SUNDER, V. S. 1978 Bounded Integral Operators on L ${ }^{2}$ Spaces. Berlin: Springer.

Hansen, E. H. 1975 A Table of Series and Products. Englewood Cliffs, NJ: Prentice-Hall.

HENRICI, P. 1979 Fast Fourier methods in computational complex analysis. SIAM Rev. 21, 481-527.

HenricI, P. 1986 Applied and Computational Complex Analysis vol. 3. New York: Wiley.

HoIDN, H.-P. 1983 Die Kollokationsmethode angewandt auf die Symmsche Integralgleichung. PhD Thesis, ETH Zürich.

Hough, D. M. 1990 User's guide to COMFPACK. IPS Research Report No 90-11, ETH Zürich.

HsiaO, G. C., KopP, P., \& Wendland, W. L. 1980 A Galerkin collocation method for some integral equations of the first kind. Computing 25, 89-130.

KRESS, R. 1989 Linear Integral Equations. Berlin: Springer.

LAmp, U., Schleicher, K. T., \& Wendland, W. L. 1985 The fast Fourier transform and the numerical solution of one-dimensional boundary integral equations. Numer. Math. 47, 15-38.

Lehman, R. S. 1957 Development of the mapping function at an analytic corner. Pacific J. Math. 7, 1437-1449.

LEHMAN, R. S. 1959 Developments near an analytic corner of solutions of elliptic partial differential equations. J. Math. Mech. 8, 727-760.

LUKE, L. 1978 Special Functions and their Approximations. New York: Academic.

MCLEAN, W. 1986 A spectral Galerkin method for a boundary integral equation. Math. Comput. 47, 597-607.

Mclean, W., Prössdorf, S., \& Wendland, W. L. 1989 Pointwise error estimates for the trigonometric collocation method applied to singular integral equations and periodic pseudodifferential equations. J. Int. Equations Applic. 2, 597-607.

REICHEL, L. 1984 On the determination of boundary collocation points for solving some problems for the Laplace operator. J. Comput. Appl. Math. 11, 175-196.

REICHEL, L. 1986 A fast method for solving certain integral equations of the first kind with applications to conformal mapping. J. Comput. Appl. Math. 14, 124-142.

REIFENBERG, M. 1997 Numerical solution of an integral equation for conformal mapping by means of attenuation factors. PhD Thesis, University of Fribourg, Switzerland.

SARANEN, J. \& SlOAN, I. H. 1992 Quadrature methods for logarithmic kernel integral equations on closed curves. IMA J. Numer. Anal. 12, 167-187.

SCHLEIFF, M. 1968 Über Näherungsverfahren zur Lösung einer singulären linearen Integrodifferentialgleichung. Z. Angew. Math. Mech. 48, 477-483.

SCHUMAKeR, L. L. 1981 Spline Functions: Basic Theory. New York: Wiley.

SLOAN, I. H. 1992 Error analysis of boundary integral methods. Acta Numer. 1, 287-339.

SLOAN, I. H. \& BURN, B. J. 1992 An unconventional quadrature method for logarithmic-kernel integral equations on closed curves. J. Int. Equations Appl. 4, 117-151.

Stenger, F. \& Schmidtlein, R. 1998 Conformal maps via sinc methods. Research Report, University of Utah.

YAN, Y. \& SLOAN, I. H. 1988 On integral equations of the first kind with logarithmic kernels. $J$. Int. Equations Applic. 1, 549-579. 